\title{
Displacement cascades and defects annealing in tungsten, Part I: defect database from molecular dynamics simulations
}

\author{
Wahyu Setyawan ${ }^{1, *}$, Giridhar Nandipati ${ }^{1}$, Kenneth J. Roche ${ }^{1,2}$, Howard L. Heinisch ${ }^{1}$, Brian D. Wirth ${ }^{3,4}$ and Richard J. Kurtz ${ }^{1}$ \\ ${ }^{1}$ Pacific Northwest National Laboratory, Richland, WA 99352, USA \\ ${ }^{2}$ Department of Physics, University of Washington, Seattle, WA 98195, USA \\ ${ }^{3}$ Department of Nuclear Engineering, University of Tennessee, Knoxville, TN 37996, USA \\ ${ }^{4}$ Oak Ridge National Laboratory, Oak Ridge, TN 37831, USA
}

\begin{abstract}
Molecular dynamics simulations have been used to generate a comprehensive database of surviving defects due to displacement cascades in bulk tungsten. Twenty-one data points of primary knock-on atom (PKA) energies ranging from $100 \mathrm{eV}$ (sub-threshold energy) to $100 \mathrm{keV}\left(\sim 780 \times E_{d}\right.$, where $E_{d}=128 \mathrm{eV}$ is the average displacement threshold energy) have been completed at $300 \mathrm{~K}$, $1025 \mathrm{~K}$ and $2050 \mathrm{~K}$. Within this range of PKA energies, two regimes of power-law energy-dependence of the defect production are observed. A distinct power-law exponent characterizes the number of Frenkel pairs produced within each regime. The two regimes intersect at a transition energy which occurs at approximately $250 \times E_{d}$. The transition energy also marks the onset of the formation of large self-interstitial atom (SIA) clusters (size 14 or more). The observed defect clustering behavior is asymmetric, with SIA clustering increasing with temperature, while the vacancy clustering decreases. This asymmetry increases with temperature such that at $2050 \mathrm{~K}\left(\sim 0.5 T_{m}\right)$ practically no large vacancy clusters are formed, meanwhile large SIA clusters appear in all simulations. The implication of such asymmetry on the long-term defect survival and damage accumulation is discussed. In addition, $<100>\{110\}$ SIA loops are observed to form directly in the highest energy cascades, while vacancy $<100>$ loops are observed to form at the lowest temperature and highest PKA energies, although the appearance of both the vacancy and SIA loops with Burgers vector of $<100>$ type is relatively rare.
\end{abstract}

Keywords: fusion, tungsten, irradiation damage, displacement cascade, molecular dynamics, loops

\section{Introduction}

One of the challenging issues in fusion materials is determining the effects of neutron damage in tungsten plasma facing components under fusion relevant conditions. The challenge stems from the unavailability of a high flux 14-MeV fusion neutron source. Therefore, development of models to predict neutron damage through computer simulations is essential for designing and interpreting experiments performed in fission reactors and linear plasma devices. An example is the extensive effort to develop an open-source code, XOLOTL-PSI [1], to simulate plasma-surface interactions with tungsten. Being a multiscale simulation tool, XOLOTL-PSI requires input from lowerlevel atomistic simulations. In this effort, a recently formulated $\mathrm{W}$ potential [2] is adopted to gather knowledge of atomistic processes for XOLOTL-PSI. This paper is concerned with the development of a database of primary defect states of radiation damage in tungsten. Along the way, several discoveries are made including a transition energy that separates different regimes of energy associated with different defect survival mechanisms, as well as asymmetric defect clustering behavior (i.e. strong differences in self-interstitial atom vs. vacancy clustering).

*Corresponding author. Tel.: +1(509)371-7692, fax +1(509)375-3033, Email address: wahyu.setyawan@pnnl.gov

Preprint submitted to Journal of Nuclear Materials
Cascade damage databases at 300, 1025 and $2050 \mathrm{~K}$ are compiled. A systematic study of cascade energy ranging from $100 \mathrm{eV}$ to $100 \mathrm{keV}$ is performed at each temperature to better sample the full recoil energy spectrum of the primary-knockon atom (PKA) due to fusion neutrons. As such, this paper presents the most comprehensive molecular dynamics (MD) database of cascade simulations in tungsten to date $[3,4,5,6]$. The range of PKA energies covers sub-threshold, low- and high-energy regimes. Data at $2050 \mathrm{~K}$ allows the investigation of the influence of temperature on defect production and morphology to be performed up to 0.5 of the melting temperature, $T_{m}$. With this comprehensive set of PKA energies and temperatures, we are able to reveal the profound effect of temperature on the asymmetry of defect clustering.

We have performed both displacement cascade and defect annealing simulations in tungsten. The results are presented in two parts. This paper reports on the primary damage production. Molecular dynamics simulations, with the LAMMPS code [7], have been used to perform the cascade simulations. A companion paper focuses on modeling the long-term evolutions of the resulting defect structures using an object kinetic Monte Carlo (OKMC) technique. That research involved both the development of modular and versatile OKMC software, kSOME and the simulations to determine the fate of the cascade dam- 
age, both of which are presented in a companion paper as Part II [8].

\section{Methods}

The $\mathrm{W}$ potential used in this study [2] is derived from the Ackland-Thetford potential [9]. A new modification hardens the potential at short distances for radiation damage simulations and improves the potential at distances of relevance to self-interstitial configurations. Prior to cascade simulations, all systems are thermalized for 30 ps at zero pressure with a NoséHoover thermostat to obtain a proper distribution of atom positions and velocities. A cascade is initiated by giving a random PKA near the center of the simulation cell an initial velocity with a random direction. The PKA initial kinetic energy is denoted as $E_{M D}$. The cascade is simulated using a microcanonical ensemble for the first $\sim 10 \mathrm{ps}$ and subsequently thermostated in a constant volume so that the target temperature is achieved within the next 1 ps. A typical total simulation time is $\sim 50 \mathrm{ps}$. The effect of the thermostat setting on defect counting and clustering is negligible. An adaptive time step is used, allowing a maximum displacement of $0.005 \AA$ per step. Sufficiently large simulation cells are used to ensure no displaced atoms cross the periodic boundaries. Displaced atoms are defined as those beyond $0.3 a_{0}$ of any lattice site, where $a_{0}$ is the lattice constant at the corresponding temperature. The calculated lattice constants at 300,1025 and $2050 \mathrm{~K}$ are $3.17,3.18$ and $3.22 \AA$, respectively. Note that these temperatures represent homologous temperatures of $0.07,0.25$ and 0.5 for the potential, respectively.

As usual, a self-interstitial-atom (SIA) or a vacancy is determined from the Wigner-Seitz cells occupancy. The list of PKA energies, simulation cell sizes and the number of simulation runs is presented in Table 1. When appropriate, the analysis presented in this paper is given as a function of PKA energy normalized by the average displacement threshold energy, $E_{d}$. The conversion to the reduced energies is included in Table 1 for convenience.

\section{Results}

\subsection{Defect production}

The number of surviving Frenkel pairs, $N_{F}$, is plotted in Figure 1 a as a function of reduced energy defined as $E^{*} \equiv E_{M D} / E_{d}$, where the calculated average displacement threshold energy is $E_{d}=128 \mathrm{eV}$ [2]. The value of $E_{d}$ signifies the energy at which the probability to create a Frenkel pair is $P_{F}=0.5$. In each of the simulations with $E^{*}<2, N_{F}$ is either zero or one, but never $>1$. Therefore, in this energy regime, $P_{F}=N_{F}$, i.e. $P_{F}$ can be directly read from the $N_{F}$ curve. Comparing the value of $N_{F}$ at $E^{*}=1$ for different temperatures, we observe the following. At $300 \mathrm{~K}$, the value of $N_{F}$ is $\sim 0.5$, indicating that $E_{d}$ remains unchanged for a relatively wide range of low temperatures from $10 \mathrm{~K}$ to $300 \mathrm{~K}$ in tungsten. It is known that a thermally activated Frenkel pair may recombine hence increasing the effective value of $E_{d}$. This phenomenon is likely responsible for the
Table 1: List of PKA energies $\left(E_{M D}\right)$, simulation cells (cubes with side length $L$ expressed in lattice constant $a_{0}$ ) and the number of simulations $\left(N_{r}\right)$.

\begin{tabular}{cccccc}
\hline \hline $\begin{array}{c}E_{M D} \\
(\mathrm{keV})\end{array}$ & $E_{M D} / E_{d}$ & $\begin{array}{c}L \\
a_{0}\end{array}$ & $\begin{array}{c}N_{r} \\
300 \mathrm{~K}\end{array}$ & $\begin{array}{c}N_{r} \\
1025 \mathrm{~K}\end{array}$ & $\begin{array}{c}N_{r} \\
2050 \mathrm{~K}\end{array}$ \\
\hline 0.1 & 0.78 & 15 & 40 & 40 & 40 \\
0.15 & 1.17 & 15 & 40 & 40 & 40 \\
0.2 & 1.56 & 15 & 40 & 40 & 40 \\
0.3 & 2.34 & 15 & 40 & 40 & 40 \\
0.5 & 3.91 & 20 & 20 & 20 & 20 \\
0.75 & 5.86 & 20 & 20 & 20 & 20 \\
1 & 7.81 & 30 & 20 & 20 & 20 \\
1.5 & 11.72 & 30 & 20 & 20 & 20 \\
2 & 15.63 & 30 & 20 & 20 & 20 \\
3 & 23.44 & 30 & 20 & 20 & 20 \\
5 & 39.06 & 40 & 20 & 20 & 20 \\
7.5 & 58.59 & 40 & 20 & 20 & 20 \\
10 & 78.13 & 50 & 20 & 15 & 15 \\
15 & 117.19 & 50 & 20 & 15 & 15 \\
20 & 156.25 & 64 & 20 & 15 & 15 \\
30 & 234.38 & 64 & 20 & 15 & 15 \\
40 & 312.50 & 64 & 15 & 15 & 15 \\
50 & 390.63 & 80 & 15 & 15 & 15 \\
60 & 468.75 & 80 & 15 & 15 & 15 \\
75 & 585.94 & 100 & 15 & 15 & 15 \\
100 & 781.25 & 120 & 20 & 20 & 20 \\
\hline & $300 \mathrm{~K}$ & $1025 \mathrm{~K}$ & $2050 \mathrm{~K}$ & & \\
$a_{0}(\AA)$ & 3.167 & 3.184 & 3.216 & & \\
\hline \hline
\end{tabular}


decrease of $N_{F}$ to 0.3 at $1025 \mathrm{~K}$. On the other hand, temperature causes thermal expansion and consequently can slightly weaken the atomic bonds. To illustrate, at 300,1025 and 2050 $\mathrm{K}$, the lattice constant is respectively $3.17,3.18$ and $3.22 \AA$, and the potential energy is $-8.86,-8.75$ and $-8.59 \mathrm{eV} /$ atom. The weaker the atomic bonds, the easier it is to create a defect. This bond weakening could explain the increase of $N_{F}$ back to 0.5 at $2050 \mathrm{~K}$. Note that more simulations (40 runs) are performed in this sub-threshold regime to increase the statistical robustness of the results. The standard error of $N_{F}$ in this regime is 0.08 , which indicates that the above variation of $N_{F}$ is statistically meaningful. The statistics are included in the Supplemental information [10].

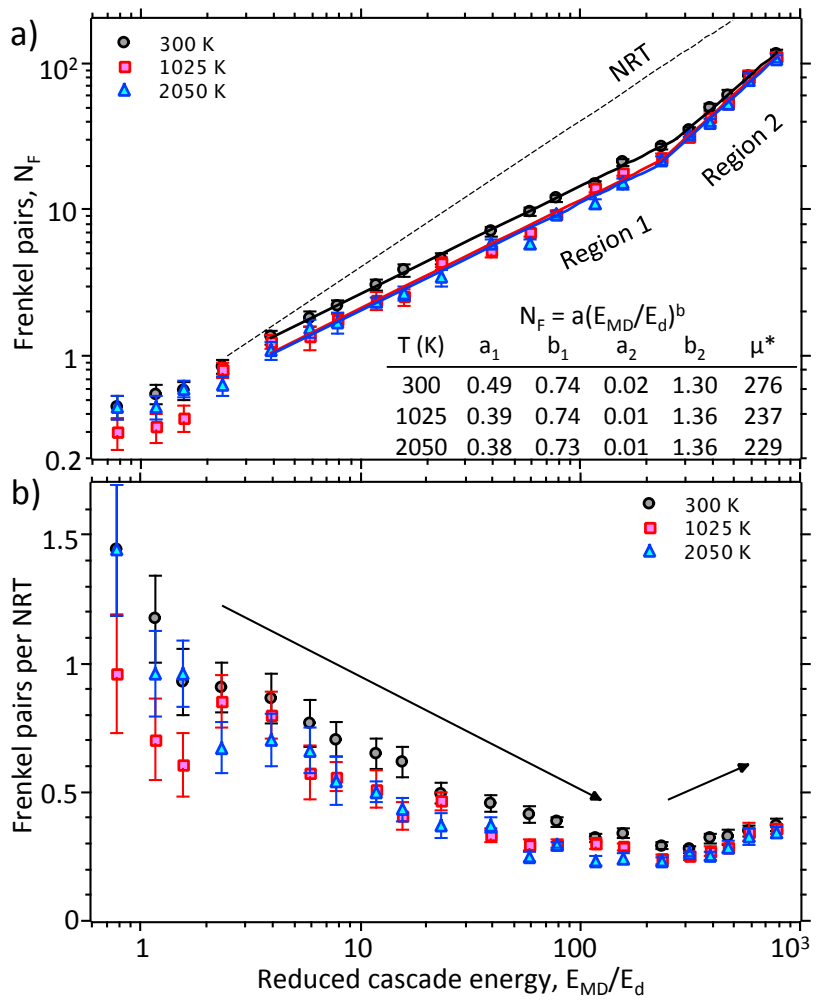

Figure 1: a) Plots of $N_{F}$ vs. $E_{M D} / E_{d}$ and power-law fits revealing two energy regimes with the transition occurring at a reduced energy $\mu^{*}$, b) Ratio of surviving MD Frenkel pairs to the number of displacements obtained from the NRT model.

A steady creation of defects occurs for reduced damage energy $E^{*} \geq 4$. For this reason, only data points in this range are included in the power-law fits. The data naturally separates into two regimes, each of which is well fit as shown in Figure 1a. The lower- and higher-energy regimes are labeled Region 1 and Region 2, respectively. A linear regression is employed for the fitting. The highest energy data in Region 1 and the lowest energy data in Region 2 for the fitting are determined by minimizing the total residual of the two fit lines. The intersection of the fit lines is defined as the transition energy, $\mu$. The coefficients of the power-law curves and the reduced transition energy, $\mu^{*} \equiv \mu / E_{d}$, are included in Figure 1a. Setyawan et al. showed that in body-centered cubic (bcc) $\mathrm{Cr}, \mathrm{Fe}, \mathrm{Mo}$ and $\mathrm{W}$ near room temperature the transition occurs at $\mu^{*} \sim 280$ to 380 [11].

In Figure 1a, the Norgett-Robinson-Torrens (NRT) displacement model, $N_{F}=0.4 E^{*}$, is plotted for comparison. It is evident that the $N_{F}$ curves obtained from MD simulations deviate from the NRT model. In Region 1, sublinear defect creation is observed and the slopes are practically invariant with respect to temperature $(b=0.74)$. On the other hand, a superlinear behavior occurs in Region 2, for which the slope increases slightly from 1.30 at $300 \mathrm{~K}$ to 1.36 at both $1025 \mathrm{~K}$ and $2050 \mathrm{~K}$. The defect production occurring during the MD simulations is significantly smaller than that predicted with the NRT model. Figure $1 \mathrm{~b}$ shows the defect survival efficiency as a function of energy for the three temperatures. The plots exhibit a V-shape curve, reflecting this sublinear and superlinear behavior. Arrows in Figure $1 \mathrm{~b}$ show this trend, with the minimum ratio of defect production from the MD simulations relative to the NRT model is found to be $\sim 0.25$.

\subsection{Defect clustering}

Generally, clustering of defects is calculated with a relatively strict connectivity cutoff limited to the first (NN1) or the second (NN2) nearest neighbor distance. In MD cascade simulations, the distribution of defects in space is determined by the cascade process and migration of the defects within the simulation time. Self-interstitial atoms are mobile and tend to cluster due to mutual attraction between them. Therefore, it is appropriate to consider the cutoff based on the clustering behavior of an interstitial pair (di-interstitial). We find that the most stable dumbbell is a $<111>$ dumbbell and the most stable configuration for a di-interstitial is two parallel $<111>$ dumbbells separated at the third nearest neighbor distance (NN3). These results are consistent with a density functional theory (DFT) calculation [12].

Unlike a di-interstitial, a DFT calculation shows that a divacancy is unstable [13] (the strongest repulsion being at the NN2 separation). Note that a three-vacancy cluster is stable and can attract additional vacancies. Nevertheless, a vacancy is practically immobile within typical MD time scales. Therefore, it is desirable to use a cutoff that corresponds to potential for clustering if the cascades were to be annealed for a longer time in OKMC simulations. Such a cutoff can be deduced from a sparse radial distribution function, $g_{s}(r)$, defined as

$$
\begin{aligned}
& g_{s}(r) \equiv \frac{2 !(N-2) !}{N !} \sum_{i=1}^{N-1} \sum_{j>i}^{N} \delta\left(r_{i j}-r\right) \\
& I_{s}(r)=\int_{0}^{r} g_{s}(x) d x, \quad I_{s}(\infty)=1
\end{aligned}
$$

where $N$ is the number of defects of interest. The notation $g_{s}(r)$ is similar to the radial distribution function $g(r)$ but for a sparse distribution (hence the subscript $s$ ). With the above definition, a quantity $(N-1) g_{s}(r)$ denotes the average number of neighboring point defects at a distance $r$ from a defect. The plots of 
$\left(N_{F}-1\right) g_{s}(r)$ for SIAs and vacancies from the $10-\mathrm{keV}$ cascades are shown in Figure 2. Note that the plots for all energies in Region 1 are similar to those depicted in Figure 2. It will be shown later that SIA loops and vacancy loops can form at energies in Region 2. The formation of such loops enhances the peak at NN1 of the $\left(N_{F}-1\right) g_{s}(r)$ plots. Therefore, the plots from Region 1 are more appropriate to study the clustering tendency of individual point defects.
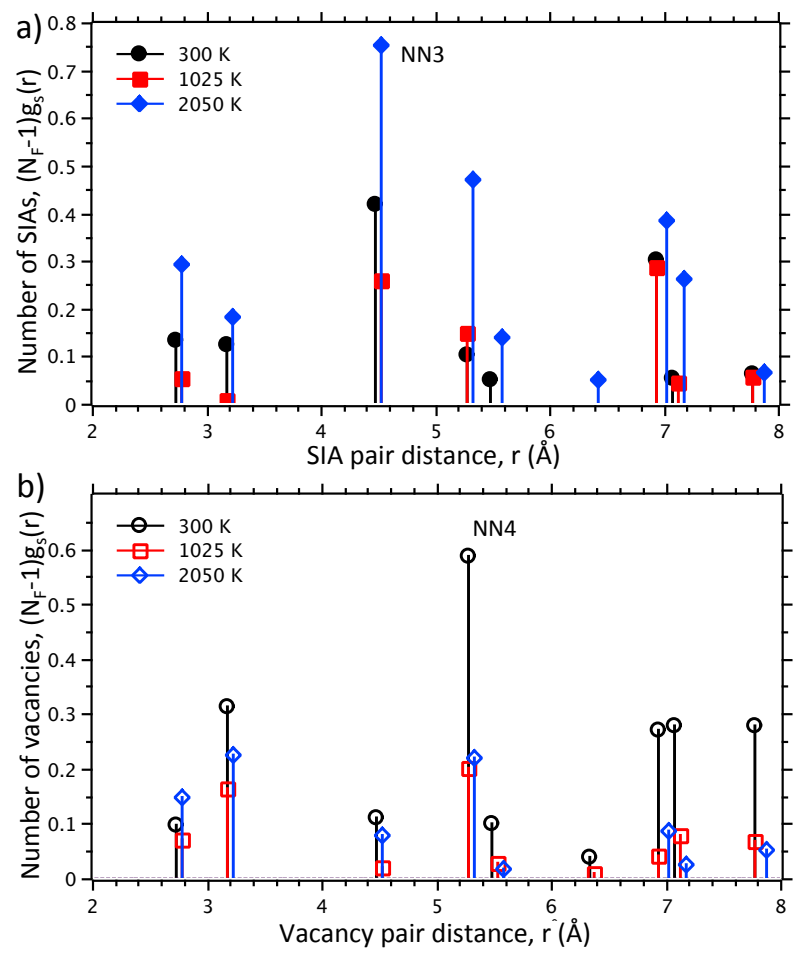

Figure 2: Sparse radial distribution function, $\left(N_{F}-1\right) g_{s}(r)$ (see Equation 1), for a) SIAs and b) vacancies. These distributions are taken from the $10-\mathrm{keV}$ cascades and reveal a dominant correlation at the third (NN3) and the fourth (NN4) nearest neighbor distance for di-interstitials and di-vacancies, respectively.

In Figure 2, a dominant peak at NN3 is found for the SIAs, further reflecting the stable configuration of a di-intersitial as described before. On the other hand, for vacancies, a dominant spatial correlation is found at the fourth nearest neighbor distance (NN4). The correlation of vacancies at NN4 was also observed for displacement cascades in Fe [14]. From this analysis, we take NN3 and NN4 as the clustering cutoff for SIAs and vacancies, respectively and use this as a default through the remainder of this article. However, for completeness, we generate another set of clustering data using a general cutoff of NN2 for both SIAs and vacancies, and this full analysis of the cluster counting data is compiled in the Supplemental information [10]. Both sets are also studied in the OKMC simulations to investigate their respective long-time evolution [8].

During the MD simulations, occasional vacancy migrations are observed, but only at $2050 \mathrm{~K}$. Such migrations are rare and do not alter the observed clustering behavior. Several graphs to illustrate the clustering trend as a function of energy and temperature are shown in Figure 3. The plotted quantities are: the fraction of clustered defects, the average size of the largest clusters $\left(S_{S I A}^{\max }\right.$ or $\left.S_{\text {vac }}^{\max }\right)$ and the number of large clusters per cascade $\left(C_{\text {SIA }}^{\text {large }}\right.$ or $\left.C_{\text {vac }}^{\text {large }}\right)$. To calculate $S_{\text {SIA }}^{\max }$ or $S_{\text {vac }}^{\max }$, the size of the largest cluster from each simulation run is taken and then averaged over the number of runs. The minimum size of a cluster that can be resolved in a transmission electron microscope studies is a debatable matter. For the sake of our discussion, we consider a large cluster as one with size $\geq 14$ (here, 14 signifies the sum of the first and second nearest neighbors in a tungsten lattice).

The clustered SIA fraction increases as a function of energy and exhibits a somewhat concave downward curvature. At lower temperatures up to $1025 \mathrm{~K}$, the fractions are still $<0.5$ for energies below the transition energy (shown by a vertical gray bar in Figure 3). Above the transition, the fractions are between 0.5 and 0.7 . Data at $2050 \mathrm{~K}$ shows a markedly larger fraction of clustered SIA with a value between 0.6 and 0.85 . Evidently the higher fraction of clustered SIAs at higher temperatures is due to the migration and binding properties of the SIAs. While the clustered SIA fraction exhibits a concave curve, the $S_{S I A}^{\max }$ plots show a convex curvature. Below the transition, $S_{S I A}^{\max }$ is limited to $\leq$ five atoms. However, above the transition $S_{S I A}^{\max }$ increases rapidly and reaches 35,45 and 55 atoms at $300 \mathrm{~K}, 1025 \mathrm{~K}$ and $2050 \mathrm{~K}$, respectively. When we count the number of large clusters, there are practically no large clusters below the transition, however large clusters readily form at energies above the transition (Figure 3c). This distinct increase in the probability of large SIA cluster formation is observed at all temperatures and indicates a correlation between the transition energy and the onset of large SIA cluster formation [11].

Temperature has an opposite effect on the fraction of vacancy clustering compared to SIA clustering. The fraction of clustered vacancies at $2050 \mathrm{~K}$ is about half that at the lower temperatures of $300 \mathrm{~K}$ and $1025 \mathrm{~K}$. Plots of $S_{\text {vac }}^{\max }$ show that the average size of the largest vacancy clusters reaches about 40-50 vacancies at $300 \mathrm{~K}$ and $1025 \mathrm{~K}$, but this value drops to around 10 at 2050 $\mathrm{K}$. This indicates that while large vacancy clusters are still commonly found at temperature up to $1025 \mathrm{~K}$, they are substantially reduced at $2050 \mathrm{~K}$.

To better illustrate the effect of temperature on SIA and vacancy clustering, snapshots of surviving defects from $75-\mathrm{keV}$ cascades at $300 \mathrm{~K}$ and $2050 \mathrm{~K}$ are depicted in Figures 4 and 5, respectively. Self-interstitial atoms or vacancies are plotted in gray or black dots (green or red in the online version). The label on each projected snapshot describes the particular simulation condition as follows. For example, the label 1:115/xy indicates that the snapshot is for simulation run-1 that produces $N_{F}=$ 115 and the view is projected on the xy-plane. Large clusters are labeled with their size. Note that at this energy, there are 15 simulation runs. All snapshots at $2050 \mathrm{~K}$ are shown in Figure 5 . For the $300 \mathrm{~K}$ data, only 13 out of 15 runs can be included in Figure 4 due to space limitation. At $300 \mathrm{~K}$, the snapshots show that i) large vacancy clusters appear in simulations where large 

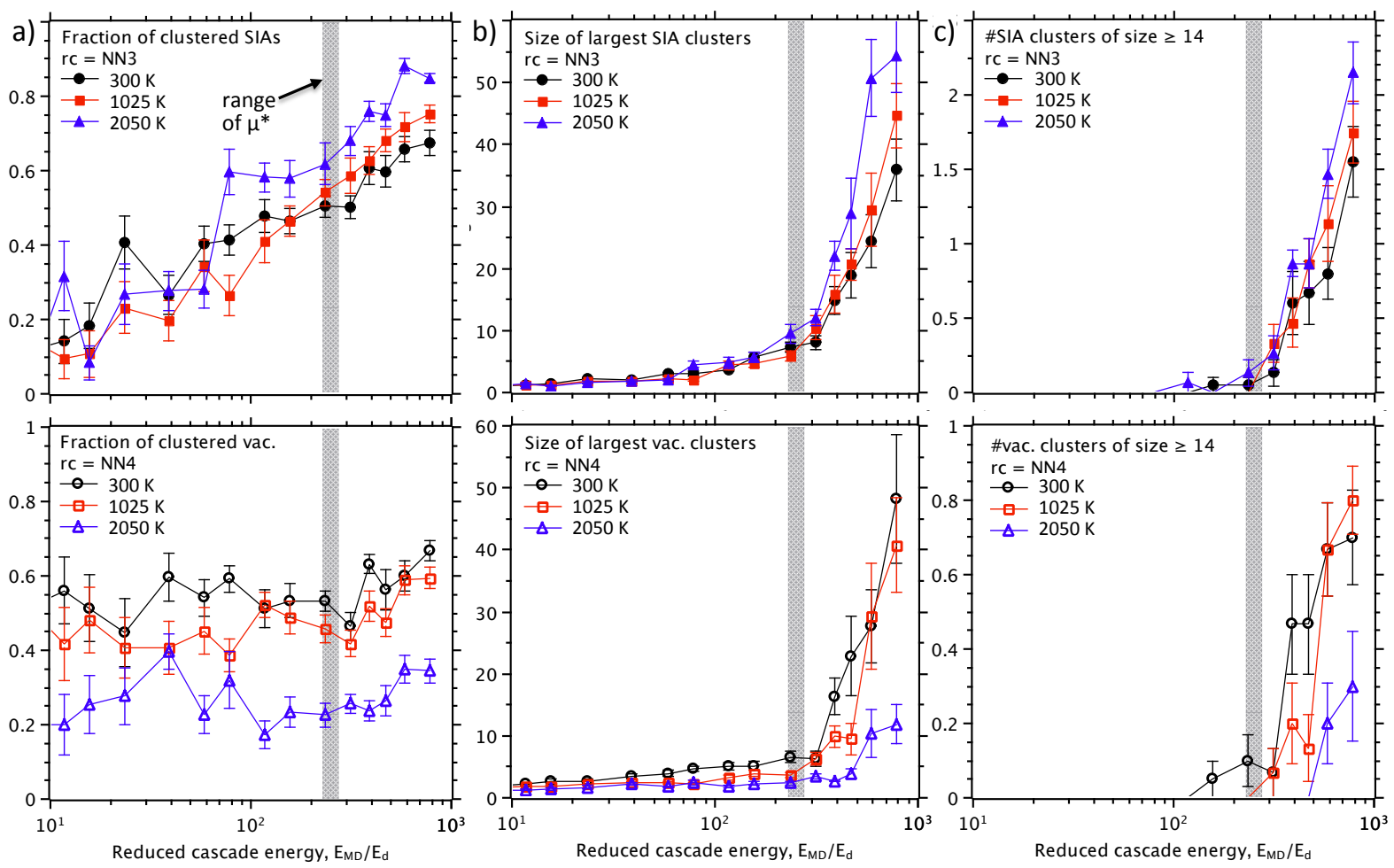

Figure 3: Energy dependence of the a) fraction of clustered defects, b) average size of the largest clusters and c) number of clusters with size $\geq 14$ per cascade calculated with clustering cutoff up to NN3 for SIAs (top panels) and NN4 for vacancies (bottom panels). 
SIA clusters also form, ii) out of 15 simulations, both large vacancy clusters and SIA clusters form in 10 runs, and iii) single SIAs and mono vacancies appear in approximately similar proportion and spatially delocalized in a similar manner. On the other hand, at $2050 \mathrm{~K}$ the snapshots show that the SIAs belonging to large SIA clusters constitute the majority of their size distribution, and in fact, large clusters form in all simulations, while for vacancies, mono vacancies constitute the clear majority of the vacancy size distribution, with large clusters forming in only 3 runs. Snapshots at $1025 \mathrm{~K}$ look similar to those at $300 \mathrm{~K}$ with both large vacancy and SIA clusters forming in 10 simulations. The $1025 \mathrm{~K}$ snapshots are only included in the Supplemental information [10] for brevity.

\subsection{Interstitial and vacancy loops}

In this study, all vacancy clusters of size $<50$ exhibit a threedimensional (3D) shape (cavity). Larger clusters can be found as either cavities or platelets (loops). From all simulations, the number of vacancy loops at 300,1025 and $2050 \mathrm{~K}$ is 13,7 and 1 , respectively. All vacancy loops appear to be $<100>\{100\}$ loops. For SIAs, clusters of size $<30$ are all found as 3D SIA clusters. Larger clusters may form 3D clusters and loops. Considering only the SIA clusters of size $\geq 30$, the partition of SIA clusters into loops and 3D clusters is given in Table 2. At 300 and $1025 \mathrm{~K}$, the majority of these clusters are found as either $1 / 2<111>\{111\}$ or $1 / 2<111>\{110\}$ loops. On the other hand, the majority of the SIA clusters at $2050 \mathrm{~K}$ are $3 \mathrm{D}$ clusters. In MD cascade simulations of bcc metals, $<100>$ SIA loops are rarely observed. In this study, several $<100>\{110\}$ SIA loops are observed at $1025 \mathrm{~K}$ and $2050 \mathrm{~K}$. There are no $<100>\{100\}$ SIA loops observed. In Table 2, several loops are identified as mixed loops. A mixed loop exhibits a $1 / 2<111>$ loop in one part and a $<100>$ loop in another part. Note that, each of these parts must constitute less than $2 / 3$ of the size for the loop to be determined as a mixed loop.

Figure 6a shows a [100](110) SIA loop of size 75 formed at $1025 \mathrm{~K}$ with a $75-\mathrm{keV}$ PKA. The atoms in dumbbell configurations are colored based on their orientation. Red atoms are part of [100] dumbbells while blue atoms are part of $<111>$ dumbbells. During the formation of this loop, $1 / 2<111>$ SIA loops were not observed. It appears that the $<100>$ SIA loop is formed directly during the cooling of the cascade melt from a collection of interstitial atoms. All $<100>$ SIA loops observed in this study have a shape of a parallelogram (or a rhombus), not a rectangle. The edges of the parallelogram are oriented along $<111>$ on one of the $\{110\}$ planes. Therefore, these edges form a set of $109.47^{\circ}$ and $\left(180^{\circ}-109.47^{\circ}\right)$ angles. Figure $6 \mathrm{~b}$ shows a mixed SIA loop, consisting of a [111̄] and a [001] loop. This loop is also formed at $1025 \mathrm{~K}$ but from a different $75-\mathrm{keV}$ cascade compared to the loop shown in Figure 6a. In Figure 6b, both of the [111] and [001] loop parts are formed at an approximately the same time during the cascade cooling. It appears that both loops maintain their Burgers vector until the end of the MD run, resulting in a mixed SIA loop. Presumably, a much longer time is needed to see whether this loop will eventually transform into a $<100>$ or a $1 / 2<111>$ loop. Stability comparison between $<100>$ and $<111>$ loops will be investigated in the future. In Fe, it was previously shown using the SEAKMC code that such transformation could occur on the microsecond time scale [15].

\subsection{Comparison with previous studies}

Björkas et al. [5] (B09) and Fu et al. [6] (F13) have also performed cascade simulations, although each used a different interatomic potential than this study [2]. We use the potential developed by Juslin et al. [2] (J13). J13 is derived from the Ackland-Thetford potential [9] (AT87). B09 is derived from the potential developed by Derlet et al. (DD07) [16]. F13 is derived from the potential developed by Dai et al. [17] (Dai06). Cascade studies in B09 and F13 were performed at $300 \mathrm{~K}$ and at several PKA energies from 0.5 to $20 \mathrm{keV}$. In B09 and F13, the clustering cutoff for SIAs is NN3 and for vacancies is NN2. Note that the cutoff for SIAs is the same as our default cutoff while that of vacancy clustering is shorter than the NN4 we used. However, we also tabulated the vacancy clustering data with NN2, for which we can make a consistent comparison.

Figure 7 shows the comparison of $N_{F}$, the fraction of clustered SIAs and the fraction of clustered vacancies. For the B09, the $N_{F}$ agrees very well with our results. This result is unexpected since B09 has a lower displacement threshold energy $(84.5 \mathrm{eV})$ than $\mathrm{J} 13(128 \mathrm{eV})$. However, this observation is consistent with the study of Fikar et al. [4] in which AT87 and DD07 produce similar $N_{F}$ even though these two potentials have different displacement threshold energies. It would be interesting to study, in the future, if $N_{F}$ remains similar at even higher energies. The clustering data above $1 \mathrm{keV}$ does show discrepancies; a larger clustered fraction of SIAs but a smaller clustered fraction of vacancies. Fikar et al. also observed the same discrepancies. It was shown that SIAs diffuse further away from the cascade core with the AT87 than with the DD07 potential. This result was attributed to the lower $<111>$ migration energy of SIAs in AT87 (26.7 meV) compared to that in DD07 (61 $\mathrm{meV}$ ). This could explain the discrepancy of the clustered fraction of SIAs. For the vacancy clusters, the migration energy of a vacancy is $1.45 \mathrm{eV}$ (AT87) and $2.05 \mathrm{eV}$ (DD07), respectively [4]. Hence, it is unlikely that this would play an important role in vacancy clustering. On the other hand, it was reported that the volume per vacancy in the cascade core is larger with DD07 than with AT87. It was reported that a larger cascade core would result in a lower fraction of clustered vacancies. The larger cascade melt region with DD07 was suggested to originate from the lower melting temperature compared to AT87.

For the F13, the clustering data for SIAs and vacancies are very similar to our results. Note in F13, the clustering data are only available for 10 and $20 \mathrm{keV}$ cascades, and the standard errors are not reported. The $N_{F}$ curve is also consistent with our results albeit with a somewhat lower value for data $\leq 5 \mathrm{keV}$. The PKA directions are random in our simulations and in B09, while the [135] direction is used exclusively in F13. The effect of the initial PKA direction on defect production is expected to be significant at lower energies, particularly near the displacement threshold regime. Therefore, it is possible that the different choices of PKA direction could account for the discrepancy of $N_{F}$ between our data and F13 at $\leq 5 \mathrm{keV}$. 


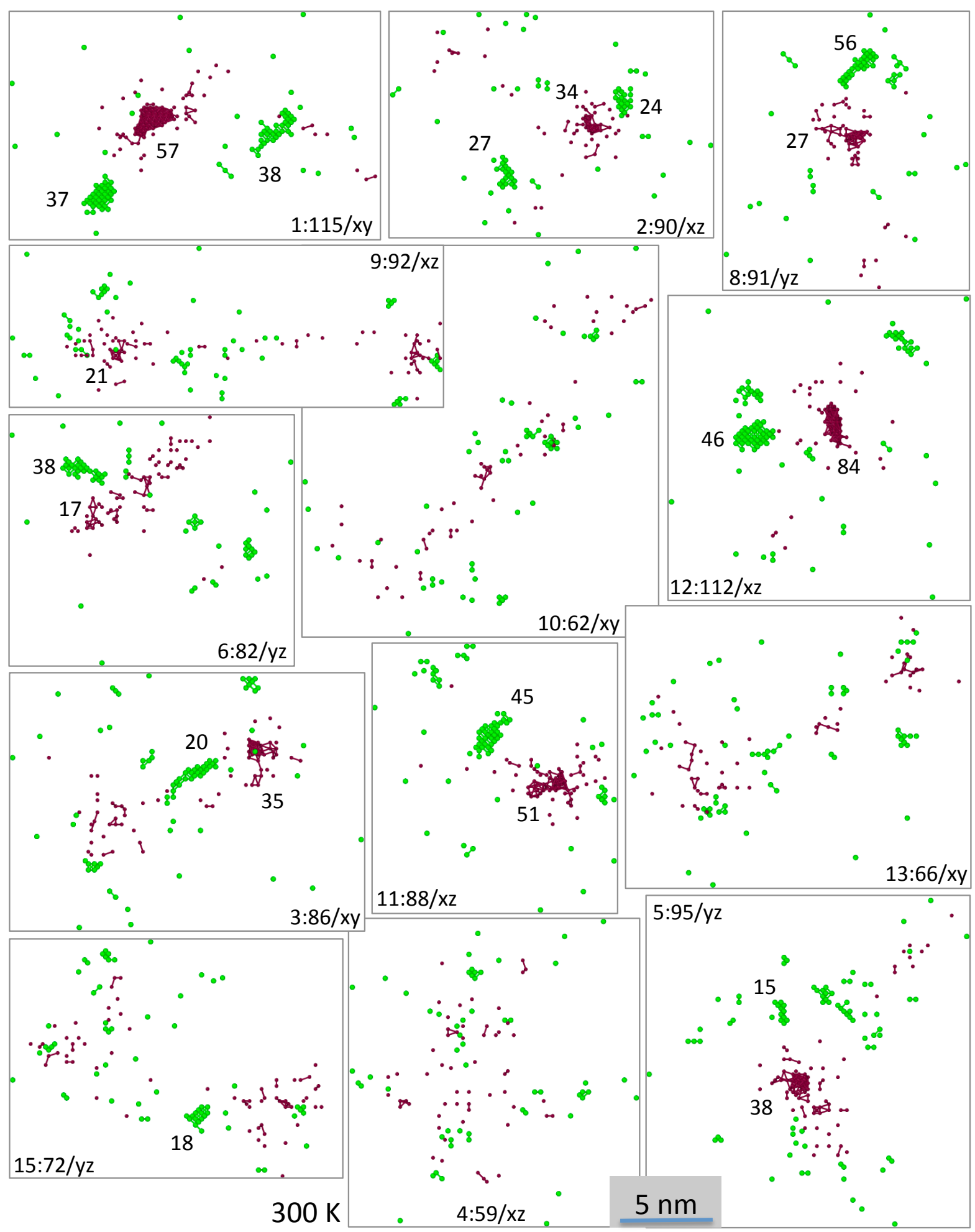

Figure 4: (color online) Snapshots of surviving SIAs and vacancies plotted in gray and black dots (green and red in online version), respectively, for $75-\mathrm{keV}$ cascade at $300 \mathrm{~K}, 13$ out of 15 runs are shown. The label's format is run: $N_{F} /$ view-projection. Clusters are determined with a cutoff up to NN3 and NN4 for SIAs and vacancies, respectively. The size of large clusters (size $\geq 14$ ) is shown. 


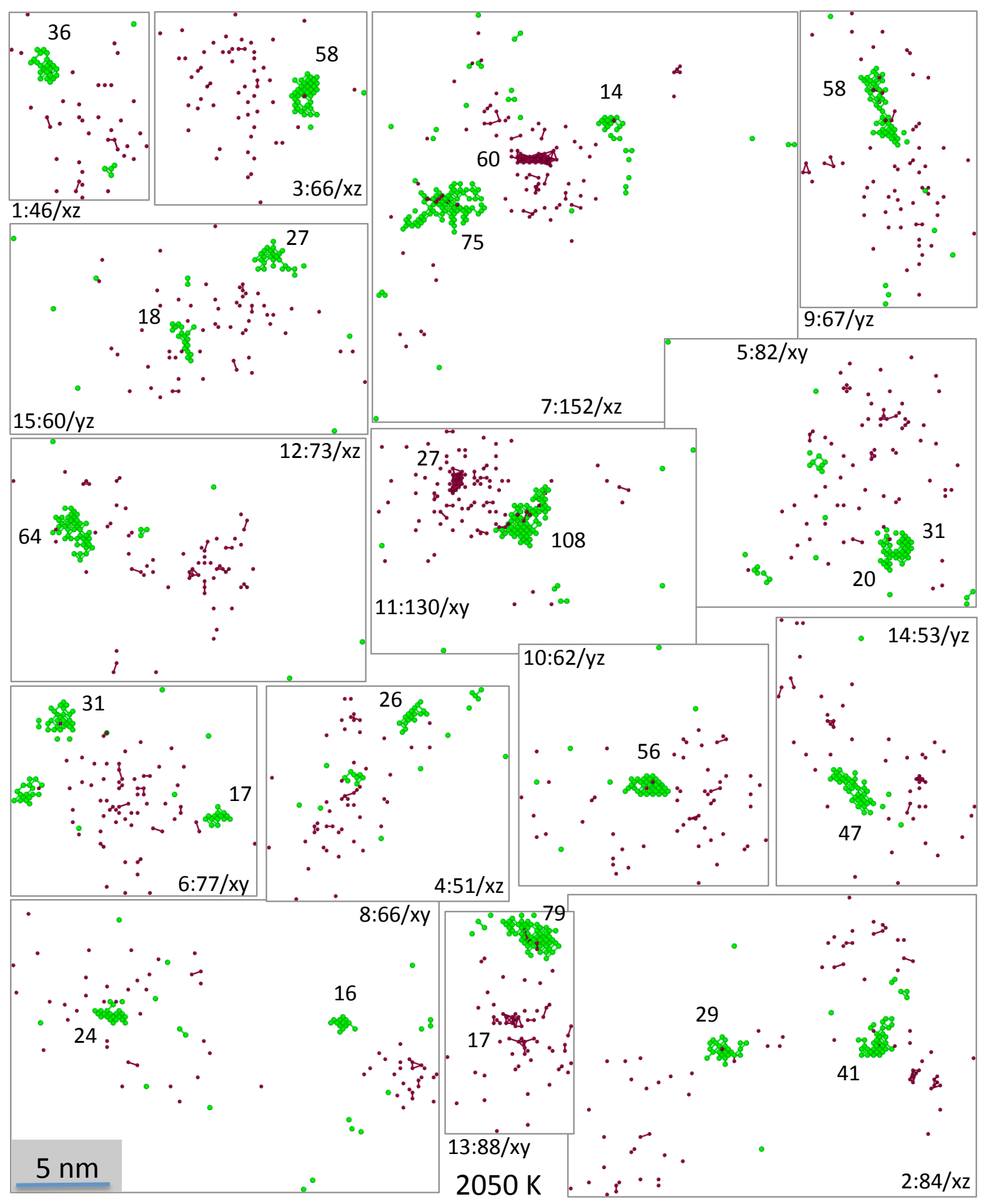

Figure 5: (color online) Snapshots of surviving SIAs and vacancies plotted in gray and black dots (green and red in online version), respectively, for $75-\mathrm{keV}$ cascade at $2050 \mathrm{~K}$, all 15 runs are shown. The label's format is run: $N_{F} /$ view-projection. Clusters are determined with a cutoff up to NN3 and NN4 for SIAs and vacancies, respectively. The size of large clusters (size $\geq 14$ ) is shown. 
Table 2: Partitioning of SIA clusters of size $\geq 30$ into loops and 3D clusters. 'Mixed loop' denotes a mixture of $1 / 2<111>$ and $<100>$ loops. '3D' denotes a 3D cluster of $<111>$ crowdions. 'Mixed 3D' denotes a mixture of $<111>$ and $<100>$ crowdions

\begin{tabular}{cccccccc}
\hline \hline & $\begin{array}{c}E_{M D} \\
(\mathrm{keV})\end{array}$ & $1 / 2<111>\{111\}$ & $1 / 2<111>\{110\}$ & $<100>\{110\}$ & Mixed loop & $3 \mathrm{D}$ & Mixed 3D \\
\hline $300 \mathrm{~K}$ & 100 & 9 & 4 & 0 & 0 & 1 & 0 \\
& 75 & 4 & 2 & 0 & 0 & 0 & 0 \\
& 60 & 2 & 1 & 0 & 0 & 1 & 0 \\
& 50 & 0 & 0 & 0 & 1 & 0 & 0 \\
& Total & 15 & 7 & 0 & 1 & 2 & 0 \\
\hline $1025 \mathrm{~K}$ & 100 & 2 & 5 & 1 & 1 & 4 & 0 \\
& 75 & 2 & 2 & 1 & 1 & 0 & 0 \\
& 60 & 0 & 1 & 0 & 0 & 0 & 0 \\
& 50 & 0 & 0 & 1 & 0 & 2 & 0 \\
& Total & 4 & 8 & 3 & 2 & 6 & 0 \\
\hline $2050 \mathrm{~K}$ & 100 & 0 & 0 & 1 & 1 & 17 & 2 \\
& 75 & 0 & 0 & 1 & 1 & 10 & 0 \\
& 60 & 0 & 1 & 0 & 0 & 3 & 1 \\
& 50 & 0 & 0 & 0 & 0 & 5 & 0 \\
& Total & 0 & 1 & 2 & 2 & 35 & 3 \\
\hline \hline
\end{tabular}

a)

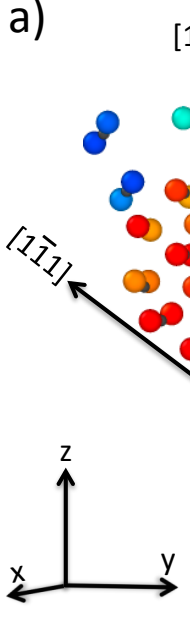

[100] loop
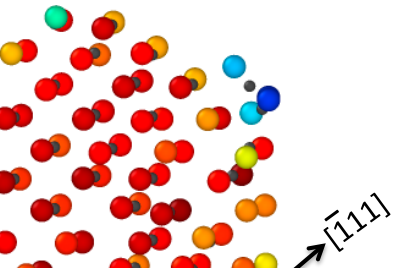

b)

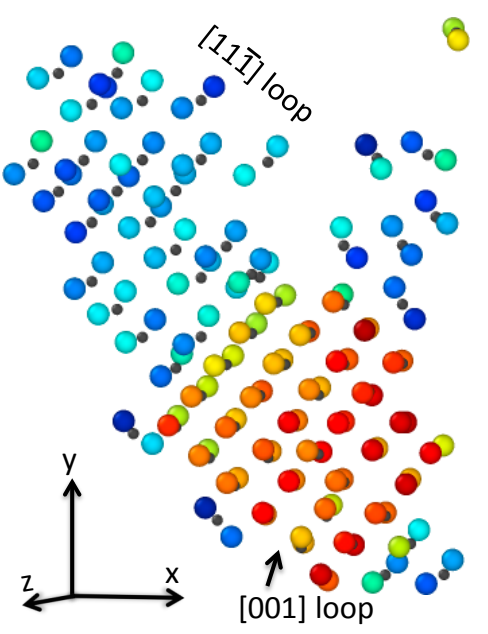

Figure 6: (color online) Snapshots of a) [100](110) SIA loop of size 75 and b) mixed SIA loop of size 68, both obtained from $75-\mathrm{keV}$ cascades at $1025 \mathrm{~K}$. 

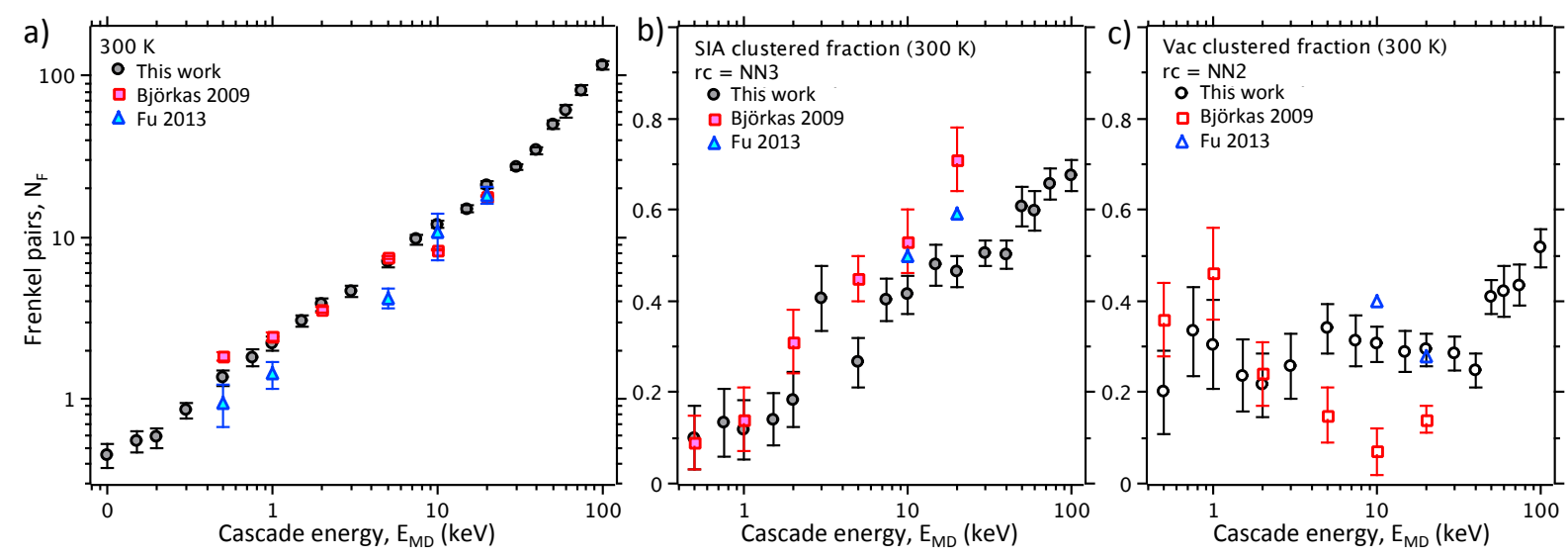

Figure 7: (color online) Comparison of a) number of surviving Frenkel pairs, b) fraction of clustered SIAs and c) fraction of vacancies obtained at $300 \mathrm{~K}$ with previous studies by Björkas 2009 [5] and Fu 2013 [6] using different interatomic potentials.

\section{Discussion}

It is commonly understood that the effect of temperature on defect production is small but clear [18]. Several factors that reduce the number of defects at higher temperature in MD simulations include higher defect mobilities, a longer lifetime of the cascade melt and the fact that the cascade itself tends to be more compact. As shown by the fit lines (see Figure 1a), the effect of temperature on the number of defects can be quantified reliably. In Region 1, the number of defects decreases $\sim 20 \%$ from $300 \mathrm{~K}$ to $1025 \mathrm{~K}$ and $\sim 5 \%$ from $1025 \mathrm{~K}$ to $2050 \mathrm{~K}$. One may consider that these variations are only a matter of annealing time scaling. However, our OKMC simulations that are described in the companion article [8] suggest that the differences remain significant if the material contains microstructures that can capture SIAs migrating away from the cascade region.

The number of defects decreases only slightly from $1025 \mathrm{~K}$ to $2050 \mathrm{~K}$. However, as described above, the amount of vacancy clustering is distinctly different between these two temperatures, i.e. large vacancy clusters are practically absent at 2050 K. Conversely, more SIA clustering occurs at higher temperature (large SIA clusters form in every simulation run at $2050 \mathrm{~K})$. The opposite effect of temperature on vacancy versus SIA clustering leads to a distinct asymmetry in defect clustering behavior and the asymmetry increases with temperature. Such an asymmetry has an important implication on the long-term survival of those defects. Indeed, the OKMC simulations show that the annealing efficiency (i.e. $N_{F}$ from OKMC simulations divided by that from MD results) is higher at $1025 \mathrm{~K}$ as compared to that at $2050 \mathrm{~K}$ [8]. The number of recombined defects during the annealing simulations depends on the product of the number of recombination events and the size of the defects that recombine. In the OKMC simulations, it is revealed that the size of the recombining defects is the more substantial factor in determining the number of recombined defects (annealing outcome). Consequently, since at 300 and $1025 \mathrm{~K}$ both large SIA and large vacancy clusters commonly form (as opposed to only large SIA clusters at $2050 \mathrm{~K}$ ), the annealing efficiency is higher

\section{at 300 and $1025 \mathrm{~K}$ than at $2050 \mathrm{~K}$.}

Through the combination of high PKA energies and temperatures, the profound effect of temperature on defect clustering asymmetry in tungsten is revealed. In the past, displacement cascade simulations in tungsten have been sparse and usually performed at $300 \mathrm{~K}$ and up to only $20 \mathrm{keV}[3,4,5,6]$. On the other hand, a comprehensive set of cascade simulations was reported for $\mathrm{Fe}$ [19] that covered $100 \mathrm{~K}, 600 \mathrm{~K}$ and $900 \mathrm{~K}$ (which is 0.5 melting temperature of $\mathrm{Fe}$ ). However, defect clustering in $\mathrm{Fe}$ is much less compared to that in $\mathrm{W}$ (presumably because $\mathrm{Fe}$ atom is far less massive than $\mathrm{W}$ ) hence any temperature effect on clustering is not readily discernable. This is why the above asymmetry and its temperature dependence is not commonly known.

Recent experimental observations suggest that large interstitial clusters might be formed within individual cascades during in situ TEM of a 150-keV self-ion irradiation of W [20]. It was reported that the interstitial-to-vacancy loop ratio was close to unity for the $1 / 2<111>$ loops, while the interstitial loops were dominant for the $<100>$ loops. The formation of such clusters is presumed to be an athermal process. Such clusters typically form within the first $0.2 \mathrm{ps}$ of the cascade development when the destructive phase occurs $[11,21]$. During this phase, the cascade energy is transferred from atom to atom much faster than phonon-mediated dissipation allows, hence supersonic shock waves form. When regions of high atomic density associated with supersonic shock waves intersect, the excess atoms from the high-density region of one shock front are deposited into the low-density region (core) associated with the other shock wave and large interstitial clusters form. This process is sometimes referred to as in-cascade cluster formation to distinguish it from the clustering process due to thermal diffusion. We have verified that in-cascade SIA cluster formation applies at all temperatures in this study. Further, we note that subsequent capture reactions of SIAs via thermal diffusion increase the size of these large clusters, as indicated by the trend of clustering data as a function of temperature. 
When a large SIA cluster forms, it induces a depletion of atoms in other regions, which potentially facilitates the formation of a large vacancy cluster. This scenario is reflected within the data at $300 \mathrm{~K}$ and $1025 \mathrm{~K}$, i.e. large vacancy clusters are obtained in simulations where large SIA clusters are also observed. Nevertheless, the formation of a large vacancy cluster eventually depends on the interplay between the recrystallization of the atoms near the core and the motion of displaced atoms in the cascade melt [22]. In other words, the controlling factor seems to be the recrystallization rate. At a higher temperature, the cascade melt persists for a longer time and the displaced atoms are also more mobile. This combination increases the chance for those displaced atoms to refill the cascade core before the atoms surrounding the core recrystallize, and this is consistent with the observation that large vacancy clusters are rarely observed in tungsten cascades at $2050 \mathrm{~K}$.

\section{Conclusions}

In conclusion, we have presented displacement cascade simulations of tungsten over a wide range of PKA energies and temperatures. The data reveal a different regime of power-law energy-dependence of the defect production above a transition energy. At the lowest temperature $(300 \mathrm{~K})$, the transition occurs at $280 \times E_{d}$, while at the highest temperature $(2050 \mathrm{~K})$, it occurs at $230 \times E_{d}$. Below the transition, the number of surviving defects, $N_{F}$, scales with energy with an exponent of 0.74 at all temperatures, while above the transition, the exponent is 1.30 at $300 \mathrm{~K}$ which increases slightly to 1.36 at $2050 \mathrm{~K}$. The more rapid increase of $N_{F}$ above the transition is associated with the formation of large SIA clusters of size 14 or more.

For temperatures up to $1025 \mathrm{~K}$, it is observed that the formation of large SIA clusters is accompanied by the formation of large vacancy clusters. The propensity for SIA clustering increases with temperature, however, the opposite is observed for vacancy clustering. This induces asymmetry in defect clustering, i.e. vacancy versus SIA clustering, which increases with temperature. A profound effect of temperature on such asymmetry is observed at $2050 \mathrm{~K}$ (i.e. 0.5 of the melting temperature) at which practically no large vacancy clusters form while large SIA clusters form in all simulations. At 300 and 1025 $\mathrm{K}$, vacancy clusters of size larger than 50 can be found as either $<100>\{100\}$ loops or cavities. For SIA clusters larger than 30 the majority are $1 / 2<111>\{111\}$ or $1 / 2<111>\{110\}$ loops at 300 and $1025 \mathrm{~K}$. However, at $2050 \mathrm{~K}, 3 \mathrm{D}$ clusters constitute the majority of the SIA clusters. We note that rare $<100>\{110\}$ SIA loops are observed at 1025 and $2050 \mathrm{~K}$.

\section{Acknowledgments}

This research has been partially supported by the U.S. Department of Energy, Office of Science, Office of Fusion Energy Sciences (\#DE-AC06-76RL0-1830), and partially supported by the U.S. Department of Energy, Office of Science, Office of Fusion Energy Sciences and Office of Advanced Scientific Computing Research through the Scientific Discovery through Ad- vanced Computing (SciDAC) project on Plasma-Surface Interactions, under Award No. DE-SC0008875. Computations were performed on Olympus cluster at Pacific Northwest National Laboratory (Fusion account). The authors would like to acknowledge the use of OVITO [23] and SCIDAVIS [24] softwares for visualization and plotting.

\section{References}

[1] XOLOTL-PSI software, http://sourceforge.net/projects/xolotl-psi/.

[2] N. Juslin, B. D. Wirth, Journal of Nuclear Materials 432 (2013) 61.

[3] N. Juslin, V. Jansson, K. Nordlund, Philosophical Magazine 90 (2010) 3581.

[4] J. Fikar, R. Schaublin, Journal of Nuclear Materials 386 (2009) 97.

[5] C. Björkas, K. Nordlund, S. Dudarev, Nuclear Instruments and Methods in Physics Research Section B 267 (2009) 3204.

[6] B. Fu, B. Xu, W. Lai, Y. Yuan, H. Xu, C. Li, Y. Jia, W. Liu, Journal of Nuclear Materials, 441 (2013) 24.

[7] S. Plimpton, Journal of Computational Physics 117 (1995) 1.

[8] A companion article to this one, G. Nandipati, W. Setyawan, H. L. Heinisch, K. J. Roche, R. J. Kurtz, B. D. Wirth, Displacement cascades and defects annealing in tungsten, Part II: object kinetic monte carlo simulation of tungsten cascade aging, http://arxiv.org/abs/1404.5247.

[9] G. J. Ackland, R. Thetford, Philosophical Magazine A 56 (1987) 15.

[10] Please see Supplemental information for additional plots, tables, and a file "defect.tgz" containing coordinates of vacancies and interstitials from the $75-\mathrm{keV}$ cascades at 300,1025 , and $2050 \mathrm{~K}$ given in LAMMPS dump file format. [please add a hypertext link to it].

[11] W. Setyawan, A. P. Selby, N. Juslin, R. E. Stoller, B. D. Wirth, R. J. Kurtz, to be published.

[12] C. S. Becquart, C. Domain, U. Sarkar, A. DeBacker, M. Hou, Journal of Nuclear Materials 403 (2010) 75.

[13] C. Becquart, C. Domain, Nuclear Instruments and Methods in Physics Research Section B: 255 (2007) 23.

[14] R. Stoller, G. Odette, B. Wirth, Journal of Nuclear Materials 251 (1997) 49.

[15] H. Xu, R. E. Stoller, Y. N. Osetsky, D. Terentyev, Physical Review Letters 110 (2013) 265503.

[16] P. M. Derlet, D. Nguyen-Manh, S. L. Dudarev, Physical Review B 76 (2007) 054107.

[17] X. D. Dai, Y. Kong, J. H. Li, B. X. Liu, Journal of Physics Condensed Matter 18 (2006) 4527.

[18] D. Bacon, F. Gao, Y. Osetsky, Journal of Nuclear Materials 276 (2000) 1.

[19] R. E. Stoller, Primary radiation damage formation, Comprehensive $\mathrm{Nu}$ clear Materials, Elsevier Ltd., Amsterdam, 2012.

[20] X. Yi, M. Jenkins, M. Briceno, S. Roberts, Z. Zhou, M. Kirk, Philosophical Magazine 93 (2013) 1715.

[21] A. Calder, D. Bacon, A. Barashev, Y. Osetsky, Philosophical Magazine 90 (2010) 863

[22] K. Nordlund, R. S. Averback, Physical Review B 56 (1997) 2421.

[23] A. Stukowski, Modelling Simul. Mater. Sci. Eng. 18 (2010) 015012.

[24] Scidavis software, http://scidavis.sourceforge.net/. 